shown that circulating galectin-3 promotes metastasis. This effect of galectin-3 is partly due to its interaction with the transmembrane mucin protein MUC1 expressed by the tumour cells, leading to clustering of MUC1 and exposure of adhesion molecules that increases cancer cell heterotypic adhesion to vascular endothelium and cancer cell homotypic aggregation to form micro-tumour emboli. We also showed that circulating galectin-3 has another, as yet unidentified, MUC1-independent action that contributes to its promotion of metastasis.

Methods Cytokine release was assessed using a protein array that includes the 36 commonest human cytokines. Galectin-3-induced adhesion of MUC1-negative human colon cancer HCT116 and melanoma ACA19- cells to microvascular lung endothelial cells (HMVECs) were assessed.

Results The presence of galectin- 3 at concentrations seen in sera of cancer patients increased the secretion of IL-6, sICAM-1, G-CSF and GM-CSF from cultured HMVECs in a galectin-3 dose- and timedependent manner. A $117.67 \pm 13.25 \%(p<0.01), 37.84 \pm 11.89 \%$ $(p<0.05), 100.33 \pm 14.55 \% \quad(p<0.01)$ and $31.47 \pm 11.36 \%(p<0.05)$ increased secretion of IL-6, sICAM-1, G-CSF and GM-CSF from HMVECs to the culture medium were seen with $1 \mu \mathrm{g} / \mathrm{ml}$ galectin- 3 after $24 \mathrm{hr}$. The culture supernatant from galectin-3-treated HMVECs increased adhesion of HCT116 (74.01 $\pm 14.33 \%, \mathrm{p}<0.05)$ and ACA19- $(43 \pm 6.67 \%, \mathrm{p}<0.05)$ cells to fresh HMVECs monolayers when compared to the supernatant from non-galectin-3 treated HMVECs. This effect was largely inhibited by the presence of a combination of neutralising antibodies against IL-6, ICAM-1, GCSF and GM-CSF or the presence of galectin-3 inhibitor lactose. Treatment of HMVECs with galectin-3 increased the expressions of HMVEC cell surface adhesion molecules integrin $\alpha_{v} \beta_{1}$, E-selectin and ICAM-1 which was largely prevented by the presence of the four neutralising anti-cytokine antibodies in combination. Serum galectin-3 concentrations were seen to be correlated $(p=0.045)$ with serum G-CSF (but not that of the other three cytokines) in colon cancer patients $(\mathrm{n}=50)$.

Conclusion Galectin-3, at concentrations found in the bloodstream of cancer patients, induces secretion of cytokines from the vascular endothelium that enhances cancer cell- endothelial adhesion as a result of up-regulation of the endothelial cell surface adhesion molecules. As cancer cell adhesion to blood vascular endothelium is an important step in metastasis, the secretion of those cytokines likely makes important contribution to galectin-3-mediated metastasis promotion.

Competing interests None declared.

\section{PM0-091 TLR 9 INHIBITION: A NOVEL TARGET OF THERAPY FOR PRIMARY LIVER CANCER}

doi:10.1136/gutjnl-2012-302514b.91

${ }^{1} \mathrm{~F}$ Mohamed, ${ }^{*}{ }^{2} \mathrm{~S}$ Minogue, ${ }^{1} \mathrm{~N}$ Shah, ${ }^{1} \mathrm{~N}$ Davies, ${ }^{1} \mathrm{~A}$ Habtesion, ${ }^{3} \mathrm{~T}$ Luong, ${ }^{4} \mathrm{~A}$ Winstanley, ${ }^{3} \mathrm{~A}$ P Dhillon, ${ }^{1} \mathrm{R}$ Mookerjee, ${ }^{1} \mathrm{R}$ Jalan. ${ }^{1}$ Department of Hepatology, University College London, London, UK; ${ }^{2}$ Centre of Molecular Biology, University College London, London, UK; ${ }^{3}$ Department of Pathology, Royal Free Hospital, London, UK; ${ }^{4}$ Department of Pathology, University College London Hospital, London, UK

Introduction Toll like receptor 9 (TLR9) is a member of the nucleotide-sensing endosomal TLR family which is critical to the innate immune defense against invading pathogens. TLR9 is activated by unmethylated $\mathrm{CpG}$ which is highly specific for bacterial DNA. Upon activation, TLR9 traffics from the endoplasmic reticulum (ER) to endosomes TLR9 signalling is inhibited by the aminoquinolone drug chloroquine.

Aims (1) assess changes in TLR9 subcellular distribution. (2) Detect any changes in the endolysosomal system. (3) Determine the effects on cell proliferation in hepatocellular carcinoma (HCC) and cholangio carcinoma cell (CC) lines upon stimulation and inhibition of TLR9 signalling in each case.
Methods Huh7D and HUCCT cells were treated with unmethylated CPG (ODN 2006) to stimulate, or chloroquine and Dynavax; IRS compound to inhibit TLR9 signalling. Cells were also treated with the TLR9 antagonist iODN. Cell growth was assessed and confocal immunofluorescence microscopy was used to determine TLR9 subcellular localisation using EEA1 and LAMP1, markers of the endolysosomal system.

\section{Results}

1. Confocal microscopy indicated a marked nuclear translocation of TLR9 in HUCCT and Huh7D when stimulated with $C p G$, while unstimulated controls showed cytoplasmic TLR9 localisation. TLR9 inhibition by $\mathrm{iODN}$ and chloroquine resulted in decreased cytoplasmic TLR9 meanwhile Dynavax treatment caused translocation of TLR9 to the perinuclear membranes.

2. Dramatic changes were also observed in the distribution of LAMP1 and EEA1, which were found to be localise to juxtanuclear punctae on TLR9 stimulation. While following inhibition they translocated to perinuclear membranes.

3. Huh7D cell counts the $C p G$ treated cells, $\mathrm{iODN}$, chloroquine and Dynavax compound were $4.5 \times 10^{5} 2.1 \times 10^{5}, 1.5 \times 10^{5}$ and $1.7 \times 10^{5}$ per $\mathrm{ml}$ respectively, compared with the untreated cells $3 \times 10^{5}$ per $\mathrm{ml}$ which indicate a significant increase in proliferation with increased TLR9 stimulation and a significant decrease with TLR9 inhibition $(p<0.03)$. In HUCCT, the $C p G$ treated cells, iODN, chloroquine and Dynavax were respectively $3.3 \times 10^{5}, 1.8 \times 10^{5}, 1.4 \times 10^{5}$ and $1.5 \times 10^{5}$ per $\mathrm{ml}$ compared with the untreated cells at $1.7 \times 10^{5}$ per $\mathrm{ml}$.

Conclusion Our study indicates that TLR9 activation increases cell proliferation whereas inhibition reduces it. Our data suggest that TLR9 may be associated with tumour proliferation and may provide a potential target for therapy in liver tumours.

Competing interests None declared.

\section{PM0-092 TLR7 EXPRESSION IS INCREASED IN HEPATOCELLULAR CANCER (HCC) AND ITS MODULATION IS ASSOCIATED WITH ALTERATIONS IN TUMOUR GROWTH: A NOVEL THERAPEUTIC TARGET}

doi:10.1136/gutjnl-2012-302514b.92

${ }^{1} \mathrm{~F}$ Mohamed, ${ }^{*} \mathrm{D}$ dhar, ${ }^{1} \mathrm{~N}$ Shah, ${ }^{1} \mathrm{~N}$ Davies, ${ }^{1} \mathrm{~A}$ Habtesion, ${ }^{3} \mathrm{~T}$ Luong, ${ }^{4} \mathrm{~A}$ Winstanley, ${ }^{3} \mathrm{~A}$ P Dhillon, ${ }^{1} \mathrm{R}$ Mookerjee, ${ }^{1} \mathrm{R}$ Jalan. ${ }^{1}$ Department of Hepatology, London, UK; ${ }^{2}$ University College London, London, UK; ${ }^{3}$ Department of Pathology, Royal Free Hospital, London, UK; ${ }^{4}$ Department of Pathology, University College London Hospital, London, UK

Introduction We have previously described upregulation of TLR9, which is mainly located in the endosomes, in human HCC and cell lines. Their inhibition or stimulation was associated with alteration in tumour growth. As TLR7 is also expressed on the endosomes we hypothesised that its expression may also be altered in HCC. The aim of the study was to determine whether TLR7 is expressed in human HCC and whether its modulation alters tumour growth.

Methods Study 1. Human tissue array platforms which included 102 cores of liver tissue (including 9 normal livers, 26 Hepatitis B and C, $25 \mathrm{HBV}$ and HCV cirrhosis and $42 \mathrm{HCC}$ ) and liver tissue obtained from a DEN/NMORE model of HCC were stained for TLR7. The scoring was performed in a blinded fashion by two individual pathologists TLR7 was scored 2 when found in $\geq 1 / 3$ of hepatocyte nucleus and 1 in $<1 / 3$. Study 2 . Human HCC cell lines (HepG2 and Huh7) were tested for the localisation of TLR7 receptors using immunoflurocense antibody, confocal microscopy and response to stimulation was tested in the presence of a specific TLR7 agonist (Imiqumoid, Invivogen) and promega proliferation assay technique. Results Study 1. TLR7 was expressed in the nucleus of hepatocytes in 34/42 HCC's with intense staining in 24; four out 25 positive in 